\title{
It is amazing what you can see if you look
}

\author{
Michael R. Pinsky
}

Published online: 27 October 2013

(C) Springer Science+Business Media New York 2013

Yogi Berra, a venerable folksy philosopher who was also a very good baseball player for the New York Yankees in their power years of the 1950s and 1960s, once said, "It is amazing what you can see if you look." This sentence has several meanings. When one looks at retrospective clinical data to define the incidence of disease or the proper use of a known effective protocol, like head of the bed elevation in ventilated patients, one is working at a disadvantage. The medical record was not specifically designed to allow such iteration of this process, which may be listed as being done but not really employed, or employed though not properly charted. These practical limitations defining incidence of use of specific protocols on available information reflect the minimal limitations of retrospective analysis. If the review also assumes restriction of defining appropriate treatment as a defined threshold value, for example a minimum of $30^{\circ}$ head of bed elevation, when subjects who are routinely placed at $25^{\circ}$ in some units or report the head of bed elevation as present or not, may be incorrectly counted as compliant or not with this therapy. Clearly, the accuracy of the findings become even less accurate and conclusions about practice made about practice patterns questionable the more assumptions are made about the data. But things can be even worse, one may study the use of a proven treatment using clinical data collected during a time when head of the bed elevation was not yet recommended or used routinely. The finding a low use of the procedure would not reflect poorly on present day compliance, only on prior lack of consideration.

\section{R. Pinsky $(\bowtie)$}

Department of Critical Care Medicine, University of Pittsburgh, 606 Scaife Hall, 3550 Terrace Street, Pittsburgh, PA 15261, USA

e-mail: pinskymr@upmc.edu
These issues are at the heart of errors in the recently published paper by Benes et al. [1] who wished to see if routine hemodynamic data from critically ill patients could be used to assess fluid responsiveness by analysis of arterial pulse pressure variation (PPV). They performed a 5-year retrospective evaluation of patients admitted with diagnosis sepsis, polytrauma, after high risk surgery or cardiac arrest. Relevant to this review, they quantified the occurrence of what they perceived to be major (sedation, mandatory ventilation and tidal volume, open chest and arrhythmia) and minor limiting factors (PEEP level, use of vasopressors and presence of arterial catheter) to the use of PPV as a diagnostic tool within the first $24 \mathrm{~h}$ after admission. In that study period 1,296 patients were hospitalized in their ICUs from which patients 549 (42.4\%) fulfilled all major criteria for applicability of dynamic variations. The authors conclude that only limited number of patients admitted for polytrauma $(51 \%)$, sepsis $(37 \%)$, after cardiac arrest $(39 \%)$ or surgical procedure $(33 \%)$ fulfill all the major criteria for use of PPV at the ICU admission. Furthermore, they conclude that the general use of PPV guided protocols for initial resuscitation seems not widely applicable because of this limitation in PPV availability or accuracy.

These conclusions from these data are regrettably unfounded and misdirected for several reasons. First, and probably most important, the author presumed that patients on high levels of positive end-expiratory pressure (PEEP) or receiving vasopressor therapy could not have their PPV define volume responsiveness. That is incorrect. Indeed our first published study on this topic in the literature was in patients with acute lung injury receiving increasing levels of PEEP [2]. The data clearly demonstrated that PPV defined the subsequent fall in cardiac output if PEEP was increased and once on increased PEEP PPV then predicted who would then increase their cardiac output in response to 
fluid loading. Thus, high levels of PEEP, when given to reverse hypoxia while not causing iatrogenic hyperinflation, do not preclude the use of PPV to predict volume responsiveness. Furthermore, our second study examined the usefulness of PPV in predicting volume responsiveness in critically ill septic shock patients, most of whom were receiving vasopressor therapy to sustain blood pressure [3]. Again the receiver operator characteristic curve for those data showed an almost perfect association between level of PPV and volume responsiveness. It was from these two studies that the PPV threshold of $>13 \%$ was proposed to reflect volume responsiveness. Similarly, Monge et al. [4] recently showed that the PPV to stroke volume variation (SVV) allowed them to not only assess volume responsiveness but also arterial elastance in septic shock patients. Specifically, while both PPV and SVV predicted volume responsiveness in their vasopressor-dependent septic shock patients, the PPV/SVV ratio also defined level of pathological vasoplegia requiring increased vasopressor therapy needed to increase blood pressure despite increased flow. Finally, Lanspa et al. [5] demonstrated that patients in septic shock who were breathing spontaneously PPV could also be use to define volume responsiveness, except that the threshold value needed increased from $13 \%$ during positive-pressure breathing to $17 \%$, with an excellent discriminatory value. When taken together these studies would return most of the patients in shock or at risk for shock in the Benes et al. [1] study into the evaluable cohort.

Although these data strongly support the increased availability of dynamic measures in the ICU to assess volume responsiveness in critically ill patients they do not really address the fundamental problem with the study by Benes et al. [1]. There was no defined protocol in their ICU for using dynamic physiological measures to assess volume responsiveness in critically patients. Physicians were not routinely making these measures and using them to guide therapy. So there was little reason to set the patients up so such measures could be made. For example, if a clinician does not suspect acute hepatic congestion or hepatic injury, they rarely measure liver enzymes over the course of treatment of critically ill patients. So a retrospective study of patients with congestion would conclude that liver enzymes are not routinely available to assess hepatic injury in ICU patients. Though those same data would mean that a retrospective chart review would not be very effective at identifying hepatic injury they would not mean that liver enzymes were not useful measure of the same hepatic injury. How can dynamic measures be any different? In the usual practice of critical care medicine non-invasive, minimally-invasive and invasive hemodynamic monitoring is the rule. But unless specifically queried only mean steady state values are sought and recorded. Once bedside caregivers start to understand the robust power that functional hemodynamic monitoring can bring to bedside diagnosis and management, the greater the chance that they will collect and use these data appropriately. Thus, the study by Benes et al. [1] is useful in showing that in the past we did not focus on such functional measures and the ability to review data retrospectively using these analyses may be limited, but it does not mean that such analyses cannot be readily made in most critically ill patients if so desired.

So we can concluded that it is amazing what you can see if you look, but you have to look with clear eyes.

Conflict of interest The author is a consultant to Edwards LifeSciences and LiDCO, both of which make hemodynamic monitoring devices needed for measuring these reported functional meausres.

\section{References}

1. Benes J, Zatloukal J, Kletecka J, Simanova A, Haidingerova L, Pradl R. Respiratory induced dynamic variations of stroke volume and its surrogates as predictors of fluid responsiveness: applicability in the early stages of specific critical states. J Clin Monit Comput. doi:10.1007/s10877-013-9524-8

2. Michard F, Chemla D, Richard C, Wysocki M, Pinsky MR, Lecarpentier Y, Teboul JL. Clinical use of respiratory changes in arterial pulse pressure to monitor the hemodynamic effects of PEEP. Am J Respir Crit Care Med. 1999;159(3):935-9.

3. Michard F, Boussat S, Chemla D, Anguel N, Mercat A, Lecarpentier Y, Richard C, Pinsky MR, Teboul J-L. Relation between respiratory changes in arterial pulse pressure and fluid responsiveness in septic patients with acute circulatory failure. Am J Respir Crit Care Med. 2000;162(1):134-8.

4. Monge MI, Gil A, Gracia M. Dynamic arterial elastance to predict arterial pressure response to volume loading in preload-dependent patients. Crit Care. 2011;15:R15.

5. Lanspa MJ, Grissom CK, Hirshberg EL, Jones JP, Brown SM. Applying dynamic parameters to predict hemodynamic response to volume expansion in spontaneously breathing patients with septic shock. Shock. 2013;39(2):155-60. 